\title{
Atividade física no lazer, capacidade aeróbia percebida e bem-estar subjetivo de acadêmicos de educação física em diferentes fases do curso
}

\author{
Physical activity in leisure, aerobic capacity perceived and subjective wellness \\ of physical education academics throughout the course \\ DOI: http://dx.doi.org/10.36453/2318-5104.2019.v17.n1.p13
}

\author{
Carlos Eduardo Vieira Meira ${ }^{1}$, Rubian Diego Andrade ${ }^{1,2}$ \\ ${ }^{1}$ Instituto de Ensino Superior da Grande Florianópolis (IESGF) \\ ${ }^{2}$ Universidade do Estado de Santa Catarina (UDESC)
}

\section{RESUMO}

Objetivo: Identificar como se comportam as variáveis, atividade física no lazer, capacidade aeróbia percebida e o bem-estar subjetivo de acadêmicos ao longo do curso de Educação Física. Método: Para esse estudo foram utilizados três instrumentos, sendo estes a Escala de Bem-estar Pessoal (PWI), Escala de Práticas no Lazer e a Escala de Capacidade Aeróbia Percebida. Resultados: Foram avaliados 115 acadêmicos (79 mulheres e 36 homens), com média de idade de 25,01 (6,2) anos. Identificouse que $87,5 \%$ dos acadêmicos foram considerados ativos fisicamente no início do curso, $95,5 \%$ no meio e $91,3 \%$ no final. Já para elevada capacidade aeróbia percebida, os resultados foram de $41,7 \%$ no início, $40,9 \%$ no meio, e $30,4 \%$ no final. Quanto ao bem-estar subjetivo, $25 \%$ no início, $22,7 \%$, no meio e $34,8 \%$ no final do curso foram classificados com alta satisfação com a vida. Conclusão: Os acadêmicos mantem-se fisicamente ativos durante todo o curso. No entanto, esses resultados não corroboraram com a variável capacidade aeróbia percebida que apresentou tendência a diminuir na comparação entre as fases. Quanto à variável de bem-estar subjetivo, permaneceu baixa ao longo do curso, com tendência de aumento no final da graduação.

PALAVRAS-CHAVE: Vida Acadêmica; Atividade Física de Lazer; Bem-estar Subjetivo; Capacidade Aeróbia Percebida.

\section{ABSTRACT}

Objective: To identify how the variables: physical activity in leisure, aerobic perceived capacity and the subjective well-being of academics during the course of Physical Education. Method: For this study, three instruments were used: the Personal Welfare Scale (PWI), Leisure Practice Scale and the Aerobic Perceived Capacity Scale. We evaluated 115 academics (79 women and 36 men), with a mean age of 25.01 (6.2) years. Results: We evaluated 115 academics ( 79 women and 36 men), with a mean age of 25.01 (6.2) years. It was identified that $87.5 \%$ of the students were considered physically active at the beginning of the course, $95.5 \%$ in the middle and $91.3 \%$ at the end. Already for high aerobic capacity perceived, the results were $41.7 \%$ at the beginning, $40.9 \%$ in the middle, and $30.4 \%$ at the end. Concerning subjective well-being, $25.0 \%$ at the beginning, $22.7 \%$ at the middle and $34.8 \%$ at the end of the course were classified with high satisfaction with life. Conclusions: Due to these results, it is suggested that, during graduation, with the achievement of knowledge about Physical Education, the academics improved their subjective perception about physical capacity and improved well-being.

KEYWORDS: Academic Life; Leisure Physical Activity; Subjective Well-being; Aerobic Perceived Capacity. 


\section{INTRODUÇÃO}

Nos últimos anos, a Atividade Física (AF) apresentou uma crescente importância no universo acadêmico e social por seus benefícios físicos, psicológicos e sociais, em consequência da sua prática (SPOHR et al., 2014; PALMA, 2017). Caracterizada por qualquer movimento do corpo em decorrência de contração voluntária dos músculos esqueléticos, a $\mathrm{AF}$ é compreendida pelas atividades realizadas no trabalho, como meio de deslocamento, como o andar a pé ou de bicicleta, nas atividades domésticas e no lazer. Esta última, praticada durante o tempo livre, ou seja, no tempo não empregado nas obrigações de trabalho e do lar, como por exemplo, exercícios físicos, esportes, caminhadas, pedaladas no parque, entre outras (CASPERSEN et al., 1985).

Nesse sentido, dentre os benefícios promovidos por esta prática, um dos principais é o fator de proteção da capacidade funcional, que pode ser compreendida como desempenho para a realização das atividades da vida diária (SPOHR et al., 2014; PALMA, 2017). Além disso, em termos fisiológicos possuir capacidade funcional adequada está associado a benefícios na densidade mineral óssea, nos fatores de risco cardiovascular, na força e resistência muscular e na saúde mental (JANSSEN et al., 2010).

Além dos benefícios fisiológicos supracitados, a prática de AF também aumenta de forma expressiva capacidade aeróbia (SOTERO et al., 2011). Esta entende-se pela capacidade máxima do corpo humano de transportar e metabolizar oxigênio durante uma $A F$, ou seja, é o máximo de oxigênio que as células de uma pessoa podem captar transportar e utilizar como fonte de energia durante uma atividade de intensidade máxima (ZAGATTO et al., 2008).

Para além das questões biológicas, componentes subjetivos como o bem-estar, também são relatados pela literatura como modificáveis com um estilo de vida ativo. De forma geral, pessoas com níveis adequados de AF possuem melhor percepção do bem-estar subjetivo (DAVIM et al., 2014; LIMA et al., 2018; MARTINS, 2015; RIGO et al., 2015). Este termo ganhou interesse com o passar dos anos por pesquisadores do mundo todo, pois é um campo de estudos que procura compreender as avaliações que as pessoas fazem de suas vidas. A avaliação é de caráter cognitivo (satisfação geral com a vida) e permite incluir, análise de expectativas futuras (DIENER et al., 2009; MARTINS, 2015; RIGO et al., 2015).

Nesse sentido, uma das questões que pode ter influência no bem-estar, no nível de AF, e por ora, na capacidade aeróbia percebida é a formação acadêmica superior. A transição da vida acadêmica para a vida profissional é um período que envolve importantes decisões (MOREIRA et al., 2013). Assim, o ingresso na mesma, repercute no bem-estar, na capacidade de concentração e memorização, e, consequentemente no seu desempenho acadêmico, interferindo em suas relações interpessoais, bem como na própria saúde, a qual é essencial para uma aprendizagem significativa e formação acadêmica de qualidade (BENAVENTE et al., 2011; REIS et al., 2016). Neste sentido, este estudo pretende identificar como se comportam as variáveis AF no lazer, capacidade aeróbia percebida e o bem-estar subjetivo de acadêmicos de Educação Física ao longo do curso.

\section{MÉTODOS}

A presente pesquisa foi caracteriza como descritiva, com abordagem quantitativa e do tipo transversal. A amostra foi do tipo não probabilística, composta por homens e mulheres acima de 18 anos, devidamente matriculados no curso de Educação Física de uma faculdade privada do município de São José (SC) (FERRARI, 2011; SILVA, 2011). O projeto foi aprovado pelo Comitê de Ética de Pesquisas em Seres Humanos da Universidade Paulista (CEPSH-UNIP), sob Parecer N ${ }^{\circ}$ 2.718.130/2018, respeitando todos os critérios de pesquisa instruído na Resolução N. 466/2012 do Conselho Nacional de Saúde. Os critérios de inclusão foram constituídos pela aceitação em participar da pesquisa por meio da assinatura do Termo de Consentimento Livre e Esclarecido (TCLE) para pesquisas com Seres Humanos, além disso, o correto preenchimento dos instrumentos, pertencer ao curso de Educação Física da instituição selecionada, ter idade acima 18 anos e acesso à mídia eletrônica. Os dados foram coletados por meio de questionários eletrônico via plataforma Google Docs. A coleta dos dados foi realizada no mês de agosto de 2018.

A amostra foi caracterizada por meio de questões referentes ao sexo (masculino e feminino) e idade (em anos). O curso foi segmentado em três grupos de acordo com semestre letivo em curso. O primeiro grupo, denominado início do curso, correspondeu do 1 으 ao 3 ㅇ semestre. O segundo, denominado meio do curso, correspondeu os alunos do 4 으

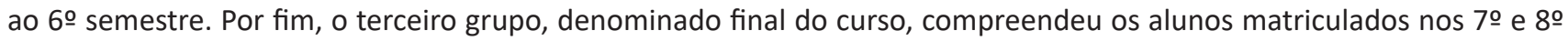
semestres. Os participantes também responderam questão referente à atividade profissional (incluindo estágio) (sim ou não), carga horária de trabalho (horas/semana) e turno de estudo (matutino ou noturno). 
Foram utilizados três questionários para a coleta de dados. O primeiro foi a Escala de Práticas no Lazer (EPL) (ANDRADE et al., 2018), validado para a população brasileira de 18 a 65 anos. $O$ instrumento é composto por questões com respostas em escala do tipo Likert de zero (nunca) a dez (sempre) pontos, na qual o participante responde sobre seu envolvimento com as práticas nos diversos conteúdos culturais do lazer - atividades artísticas, culturais, manuais, físicoesportivas, sociais, virtuais, turísticas e contemplativas (ANDRADE et al., 2018). Para atender aos objetivos da pesquisa foi utilizado apenas o domínio Físico-esportivo da EPL. Desta forma, os acadêmicos foram classificados como suficientemente ativos (>4 pontos) e insuficientemente ativos ( $\leq 4$ pontos) (ANDRADE et al., 2017).

Para avaliação do bem-estar subjetivo utilizou-se a questão "Pensando na sua própria vida e situação pessoal, qual é o seu nível de satisfação com a sua vida como um todo?" extraída do Personal Wellbeing Index (PWI) (Índice de Bemestar Pessoal) (CUMMINS et al., 2003). Este instrumento tem como intuito abordar um parâmetro subjetivo da qualidade de vida em grupos variados da população. Os autores do instrumento descrevem que esta questão tem elevado poder de predição do bem-estar subjetivo. As opções de respostas são apresentadas em uma escala Likert de dez pontos que varia de zero (0) (completamente insatisfeito) a dez (10) (completamente satisfeito). Considerou-se como elevada percepção de bem-estar a mediana (8 pontos). Desta forma, categorizou-se os acadêmicos em baixo bem-estar subjetivo e elevado bem-estar subjetivo.

O último questionário foi a Escala de Capacidade Aeróbia Percebida (Rating of Perceived Capacity) (WISÉN et al., 2002) que foi validada no Brasil por Maranhão Neto et al. (2008), e apresentou no teste e reteste um coeficiente de concordância linear de 0,78 (IC95\% 0,55-1,00) e um coeficiente de correlação interclasses de 0,8 (IC95\% 0,50-0,93). Essa escala é composta por uma lista de atividades em que o participante seleciona o que é capaz de fazer por um período mínimo de 30 minutos. Estas atividades são: ficar sentado; caminhar devagar; caminhar em um ritmo normal/ Pedalar devagar; correr devagar ("cooper")/Pedalar; correr; correr rápido/Pedalar rápido; correr muito rápido (mais do que $15 \mathrm{~km}$ ); realizar treinamento aeróbio para competição (mulheres); realizar treinamento aeróbio para competição (homens). O ponto de corte médio (mediana) para categorização desta variável foram 10 pontos. Os acadêmicos que relataram estar acima desta referência foram considerados como elevada capacidade aeróbia percebida, bem como com valores inferiores a esta mediana foram considerados com baixa capacidade aeróbia percebida.

O programa estatístico utilizado para a análise dos dados foi o Statistical Package for the Social Sciences (SPSS ${ }^{\circledR}$ ) for Windows ${ }^{\circledR}$ versão 20.0. Foram realizadas análises descritivas e inferenciais. A normalidade da distribuição dos dados foi analisada por meio do teste de Kolmogorov-Smirnov, no qual foram confirmadas a não normalidade dos dados. Para as análises de associação entre as variáveis categóricas utilizou-se o teste Qui-quadrado. Para comparação entre os grupos de variáveis contínuas foram realizados os testes Kruskal-Wallis. Para todas as análises, foi considerado nível de significância de $5 \%$.

\section{RESULTADOS}

Os dados descritivos da amostra foram apresentados na Tabela 1. Foram avaliados 115 acadêmicos (36 mulheres e 79 homens), com média de idade de 25,01 (6,2) anos, de um curso de Educação Física de uma Instituição de Ensino Superior de São José (SC). A maioria dos acadêmicos $(81,7 \%)$ no ato da coleta de dados, estavam matriculados no período noturno e relataram ter emprego ou fazer estágio (80\%). Quanto ao período do curso, $41,7 \%$ foram classificados em ingressantes, $38,3 \%$ no meio e $20 \%$ no final do curso. De acordo com os critérios e instrumentos utilizados, $8,7 \%$ foram classificados como insuficientemente ativos e $91,3 \%$ ativos fisicamente. Já com relação à capacidade aeróbia, 60,9\% relataram ter uma percepção de baixa capacidade aeróbia. Além disso, a maioria dos acadêmicos demonstrou ter uma baixa percepção subjetiva de bem-estar (73,9\%).

A Tabela 2 apresenta a diferença da pontuação das variáveis ao longo do curso. Percebe-se que nenhuma das variáveis apresentaram diferenças significativas entre os três grupos analisados (iniciantes, meio e final do curso). Apesar disso, a análise das Figuras 1, 2 e 3 apresenta a tendência no comportamento dessas variáveis entre os acadêmicos avaliados. Na Figura 1 a percepção da capacidade aeróbia praticamente manteve-se estável no início $(41,7 \%)$ e meio do curso $(40,9 \%)$, e no final do curso a percepção diminui (30,4\%). Já na Figura 2 percebe-se que o nível de acadêmicos fisicamente ativos praticamente manteve-se estável no início $(87,5 \%)$ no meio $(95,5 \%)$ e no final do curso $(91,3 \%)$. Por fim, a Figura 3 apresenta que o bem-estar subjetivo se manteve estabilizado no início $(25,0 \%)$ e no meio $(22,7 \%)$, e teve um leve aumento no final do curso (34,8\%). 
Tabela 1. Caracterização da amostra.

\begin{tabular}{|c|c|c|}
\hline Variáveis & $n$ & Valores \\
\hline \multicolumn{3}{|l|}{ Sexo (\%) } \\
\hline Masculino & 79 & 68,7 \\
\hline Feminino & 36 & 31,7 \\
\hline Idade (anos) & 115 & $25,01 \pm 6,2$ \\
\hline IMC $\left(\mathrm{Kg} / \mathrm{m}^{2}\right)$ & 115 & $24,52 \pm 2,9$ \\
\hline \multicolumn{3}{|l|}{ Status de peso (\%) } \\
\hline Eutróficos & 70 & 60,90 \\
\hline Sobrepeso & 45 & 39,10 \\
\hline \multicolumn{3}{|l|}{ Turno de estudo (\%) } \\
\hline Matutino & 21 & 18,3 \\
\hline Noturno & 94 & 81,7 \\
\hline \multicolumn{3}{|l|}{ Trabalho (\%) } \\
\hline $\operatorname{sim}$ & 92 & 80,0 \\
\hline Não & 23 & 20,0 \\
\hline Carga horária (horas/semana) & 91 & $31,76 \pm 13,5$ \\
\hline \multicolumn{3}{|l|}{ Período do curso (\%) } \\
\hline Ingressantes & 48 & 41,70 \\
\hline Meio do curso & 44 & 38,30 \\
\hline Final do curso & 23 & 20,00 \\
\hline \multicolumn{3}{|l|}{ Nível de Atividade Física (\%) } \\
\hline Insuficientemente ativos & 10 & 8,7 \\
\hline Ativos fisicamente & 105 & 91,3 \\
\hline \multicolumn{3}{|l|}{ Capacidade aeróbia percebida (\%) } \\
\hline Baixa & 70 & 60,9 \\
\hline Elevada & 45 & 39,1 \\
\hline \multicolumn{3}{|l|}{ Bem-estar subjetivo (\%) } \\
\hline Baixo & 85 & 73,9 \\
\hline Elevado & 30 & 26,1 \\
\hline
\end{tabular}

Tabela 2. Diferença das variáveis ao longo do curso.

\begin{tabular}{lcccc}
\multirow{2}{*}{\multicolumn{1}{c}{ Variáveis }} & \multicolumn{3}{c}{ Período do curso (média, dp) } & \multirow{2}{*}{-valor* } \\
\cline { 2 - 4 } & Iniciantes & Meio & Final & \\
\hline Atividade Física no Lazer & $8,46 \pm 2,4$ & $8,75 \pm 2,0$ & $8,78 \pm 2,3$ & 0,933 \\
Capacidade aeróbia percebida & $11,44 \pm 5,4$ & $10,27 \pm 4,4$ & $10,78 \pm 4,2$ & 0,785 \\
Bem-estar subjetivo & $7,31 \pm 1,7$ & $7,36 \pm 1,5$ & $7,83 \pm 1,7$ & 0,286 \\
\hline
\end{tabular}

*p-valor do teste de Kruskal-Wallis.

Este estudo teve como objetivo identificar como se comportam as variáveis, atividade física no lazer, capacidade aeróbia percebida e bem-estar subjetivo de acadêmicos em diferentes fases do curso de Educação Física. Com relação ao nível de $\mathrm{AF}$, percebeu-se uma tendência desta variável permanecer constante nas diferentes fases do curso. Foram considerados ativos fisicamente na fase inicial do curso $87,5 \%$, no meio do curso $95,5 \%$ e no final $91,5 \%$. Esses resultados corroboram com estudos de Sousa et al. (2009) e Silva et al. (2017), que avaliaram o nível de AF e qualidade de vida de acadêmicos do curso de Educação Física. Sugere-se assim, que a escolha pelo curso de Educação Física indica que os acadêmicos já possuíam uma vida fisicamente ativa. Além disso, outra hipótese levantada é que os ingressantes no curso 
possam ser em sua maioria, praticantes de desportos ou ex-atletas (KRUG et al., 2017).

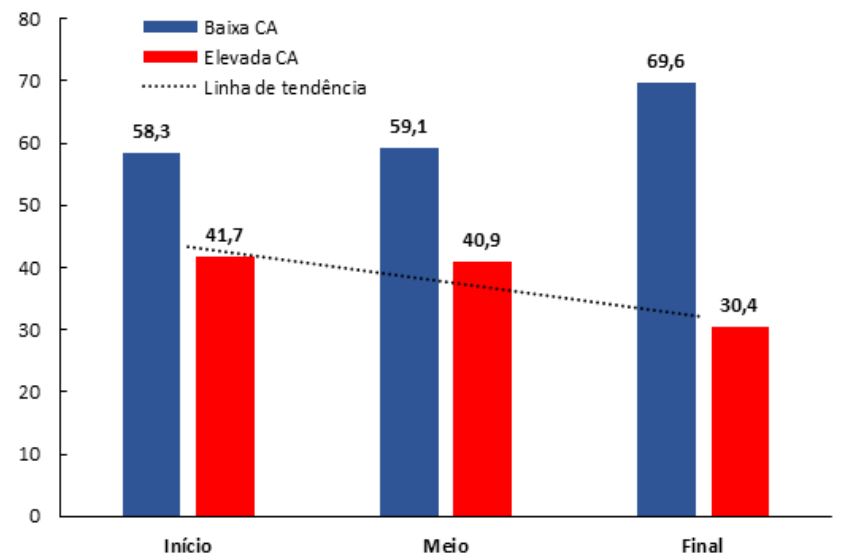

Figura 1. Capacidade aeróbia percebida nos diferentes períodos do curso.

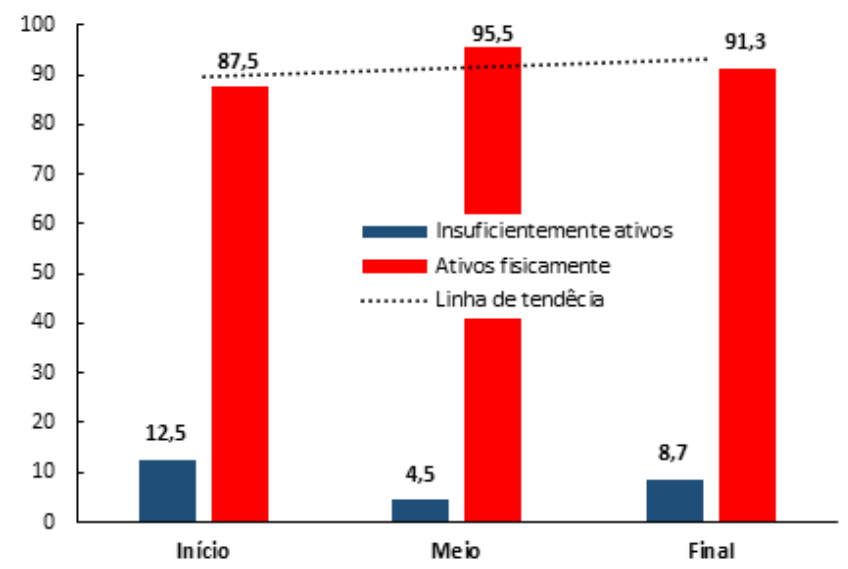

Figura 2. Nível de atividade física no lazer nos diferentes períodos do curso.

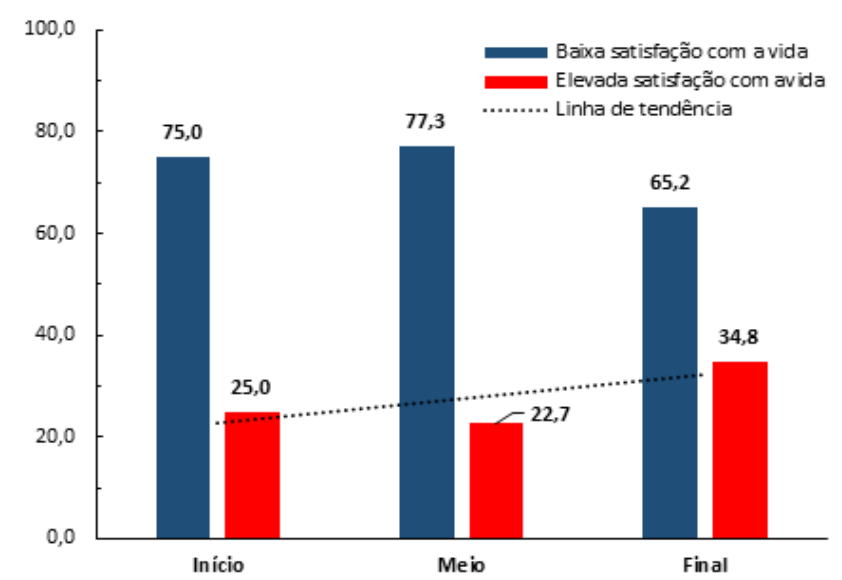

Figura 3. Bem-estar subjetivo nos diferentes períodos do curso. 
Tabela 3. Associação entre as variáveis com o período do curso, São José (SC), 2018.

\begin{tabular}{|c|c|c|c|c|}
\hline \multirow{2}{*}{ Variáveis } & \multicolumn{3}{|c|}{ Período do curso $(n, \%)$} & \multirow{2}{*}{ *p-valor } \\
\hline & Iniciantes & Meio & Final & \\
\hline \multicolumn{5}{|l|}{ Status de peso } \\
\hline Eutróficos & $32(66,7)$ & $27(61,4)$ & $11(47,8)$ & \multirow{2}{*}{0,313} \\
\hline Sobrepeso & $16(33,3)$ & $17(38,6)$ & $12(52,2)$ & \\
\hline \multicolumn{5}{|l|}{ Turno de estudo } \\
\hline Matutino & $24(43,8)$ & - & - & \\
\hline Noturno & $27(56,2)$ & $48(100,0)$ & $23(100,0)$ & \\
\hline \multicolumn{5}{|l|}{ Trabalho } \\
\hline Sim & $34(70,8)$ & $37(84,1)$ & $2(8,7)$ & \multirow{2}{*}{0,090} \\
\hline Não & $14(29,2)$ & $7(15,9)$ & $21(91,3)$ & \\
\hline
\end{tabular}

*p-valor do teste de Qui-quadrado.

Os resultados quanto ao nível de capacidade aeróbia percebida mostraram que 39,1\% dos acadêmicos tem um nível elevado desta condição. Esse resultado corrobora com o estudo de Carvalho (2016) que avaliou a capacidade aeróbia de forma indireta em acadêmicos de Educação Física por meio do teste de Cooper. Os autores identificaram que 34,7\% da amostra total apresentou capacidade aeróbia elevada. Na presente pesquisa, observou-se que ao final do curso a capacidade aeróbia apresenta uma tendência de queda (início 41,7\%, meio 40,9\% e final 30,4\%).

Sob a luz das teorias da aprendizagem e do comportamento social (BANDURA, 2008), sugere-se que esta diminuição da percepção da capacidade aeróbia elevada esteja relacionada com uma auto avaliação mais criteriosa desta capacidade nas fases finais do curso. Ainda, fazendo-se valer das teorias sociais, da mesma forma que o conhecimento muda a perspectiva do sujeito em relação ao mundo, a mesma relação talvez possa ser representada pelo conhecimento adquirido e a auto avaliação desta capacidade física. Isso pode ajudar a explicar o motivo pelo qual houve a tendência de diminuição nas diferentes fases do curso, haja vista que o nível de atividade física se manteve estável no mesmo período. Desta forma, ingressantes talvez tenham uma visão superestimada da sua capacidade aeróbia, ao passo que, na visão dos egressos, esta percepção seja diferente.

A variável bem-estar subjetivo manteve-se baixa durante o curso (início 75,0\%, meio 77,3\% e final 65,2\%). Segundo Pekmezovic et al. (2011), para os acadêmicos, a universidade é a porta de entrada para a vida profissional mais promissora no futuro, e, na prerrogativa de manter os seus empregos atuais, muitos possuem dupla jornada (trabalho e estudo). Especialmente em se tratando do contexto de uma universidade privada. Ista limita o tempo livre para a vida pessoal e para o lazer, e como consequência compromete ainda mais a qualidade de vida e bem-estar. Dos acadêmicos que participaram do estudo $80 \%$ trabalham ou fazem estágio, essa dupla jornada pode ajudar a explicar a permanecia do baixo bem-estar ao longo do curso $(73,9 \%)$. Porém, ao final do curso essa variável tem um leve aumento $(34,8 \%)$, sugerindo que a perspectiva de um futuro profissional promissor, a possibilidade de ingressar em uma nova profissão possa aumentar a "segurança com o futuro" (uma das variáveis do instrumento PWI), concomitantemente aumentando o nível de bem-estar dos acadêmicos (PEKMEZOVIC et al., 2011).

De acordo com os resultados da presente pesquisa sobre nível de AF e capacidade aeróbia percebida, sugere-se à comunidade científica novos estudos utilizando instrumentos que avaliem de forma direta essas variáveis, tendo em vista que poucos estudos avaliaram essa população desta forma. Assim, seria possível melhorar a compreensão dessas variáveis e tendo resultados mais específicos dessa população. Como limitação deste estudo, leva-se em consideração que o instrumento pelo qual foi avaliado o nível de AF, a EPL é recente. Sendo assim, há poucos estudos com o mesmo. Sendo utilizado na discussão estudos que utilizam outro instrumento para avaliação do nível de AF de forma subjetiva (IPAQ, 2005). Quanto ao nível de capacidade aeróbia percebida não foi encontrado nenhum estudo em acadêmicos, portanto utilizou-se como parâmetro de comparação um estudo que avaliou essa variável de forma indireta (CARVALHO, 2016).

No que tange o bem-estar subjetivo, a análise desta variável utilizando o instrumento por completo pode explicar em quais domínios do bem-estar estão associados a cada fase do curso (início, meio e final). Além dessas, uma limitação a ser considerada é o delineamento da pesquisa. Na presente, um estudo transversal, comparou diferentes sujeitos. Desta 
forma, o acompanhamento dos mesmos indivíduos ao longo do curso poderia melhor explicar as variáveis analisadas e estabelecendo possíveis relações de causa e efeito.

\section{CONSIDERAÇÕES FINAIS}

Os resultados do presente estudo demonstraram que os acadêmicos do curso de Educação Física se mantêm ativos fisicamente do início ao final do curso. Porém, esses resultados não corroboraram com a variável capacidade aeróbia percebida que apresentou uma tendência de queda na comparação entre as fases, sugerindo que o conhecimento adquirido ao longo o curso de Educação Física, pode ter melhorado o entendimento da mesma, tornando essa avaliação mais criteriosa.

Quanto a variável bem-estar subjetivo manteve-se baixa no início ao longo do curso com tendência de aumento no final da graduação. Sugere-se novos estudos que avaliem a variável capacidade aeróbia e atividade física de forma direta. Além disso, recomenda-se que sejam realizados novos estudos que comparem os resultados com outros cursos. Por fim, aponta-se a necessidade de estudos com delineamento longitudinal para a investigação de tais variáveis nesta população.

\section{REFERÊNCIAS}

ANDRADE, R. D.; BARBOSA, D. G.; FÉLDEN, E. P. G. Capacidade preditiva do domínio físico-esportivo da escala de práticas no lazer (EPL) para discriminação de níveis suficientes e insuficientes de atividade física. XI Congresso Brasileiro de Atividade Física e Saúde. Anais... Florianópolis, v. 22, p. 510-639, 2017.

ANDRADE, R. D.; BARBOSA, D. G.; FÉLDEN, E. P. G. Validade de construto e consistência interna da escala de práticas no lazer para adultos. Ciência \& Saúde Coletiva, Rio de Janeiro. v. 23, n. 2, p. 51928, 2018.

BANDURA, A. A evolução da teoria social cognitiva. In: BANDURA, A.; AZZI, R. G.; POLYDORO, S. (Org.). Teoria social cognitiva: conceitos básicos. Porto Alegre: Artmed, p. 15-41, 2008.

BAUMANN, M.; IONESCU, I.; CHAU, N. Psychological quality of life and its association with academic employability skills among newly-registered students from three european faculties. BMC psychiatry, Londres, v. 11, n. 1, p. 63-73, 2011.

BENAVENTE, S. B. T.; ALS, C. Respostas fisiológicas e emocionais ao estresse em estudantes de enfermagem: revisão integrativa da literatura científica. Acta Paulista de Enfermagem, São Paulo, v. 24, n. 4, p. 571-6, 2011.

CARVALHO, A. M. Nível de atividade física, capacidade cardiorrespiratória e estilo de vida em estudantes universitários. 2016. 28f. Trabalho de Conclusão de Curso (Curso de Educação Física) Universidade Federal do Rio Grande do Norte, Natal, 2016.

CASPERSEN, C. J.; POWELL, K. E.; CHRISTENSON, G. M. Physical activity, exercise, and physical fitness: definitions and distinctions for health-related research. Public Health Reports, Rockville, v. 100, n. 2, p. 126, 1985.

CUMMINS, R. A.; ECKERSLEY, R.; PALLANT, J.; VUGT, J. V.; MISAJON, R. Developing a national index of subjective wellbeing: The Australian Unity Wellbeing Index. Social Indicators Research, Boston, v. 64, n. 2, p. 159-90, 2003.

DAVIM, R. M. B.; TORRES, G. V.; DANTAS, S. M. M.; LIMA, V. M. Estudo com idosos de instituições asilares no município de Natal/RN: características socioeconômicas e de saúde. Revista LatinoAmericana de Enfermagem, São Paulo, v. 12, n. 3, p. 518-24, 2004.

DIENER, E.; SCOLLON, C. N.; LUCAS, R. E. The evolving concept of subjective well-being: The multifaceted nature of happiness. In: DIENER, E. Assessing well-being. Springer: Dordrecht, 2009.

FERRARI, E. Pesquisa Descritiva. In: SANTOS, S. G. Métodos de Pesquisa Quantitativa Aplicadas à Educação Física. Florianópolis: Tribo da Ilha. 2011. p. 81-91.

IPAQ. INTERNATIONAL PHYSICAL ACTIVITY QUESTIONNAIRE. RESEARCH COMMITTEE. Guidelines for data processing and analysis of the International Physical Activity Questionnaire (IPAQ)short and long forms, 2005. Disponível em: <https://www.researchgate.net/file.PostFileLoader. html?id=5641f4c36143250eac8b45b7\&assetKey=AS\%3A294237418606593\%401447163075131> Acessado em: 22 de outubro de 2018. 
JANSSEN, I.; LEBLANC, A. G. Systematic review of the health benefits of physical activity and fitness in school-aged children and youth. International Journal of Behavioral Nutrition and Physical Activity, Londres, v. 7, n. 1, p. 40, 2010.

KRUG, H. N.; CONCEIÇÃO, V. J. S.; KRUG, R. R.; TELLES, C.; FLORES, P. Expectativas de atuação profissional de acadêmicos ingressantes e concluintes no curso de licenciatura em educação física. Biomotriz, Cruz Alta, v. 11, n. 2, p. 84-108, 2017.

LIMA, D. F.; LIMA L. A.; MAZZARDO, O. J.; ANGUERA. M. G.; PIOVANI, V. G. S.; SILVA JUNIOR, A. P.; SILVA, M. P.; SAMPAIO, A. A. O padrão da atividade física no lazer de brasileiros idosos. Caderno de Educação Física e Esporte, Marechal Cândido Rondon, v. 16, n. 2, p. 39-49, 2018.

MARANHÃO NETO, G. A.; LEON, A. C. M. P.; FARINATTI, P. T. V. Equivalência transcultural de três escalas utilizadas para estimar a aptidão cardiorrespiratória: estudo em idosos. Cadernos de Saúde Pública, Rio de Janeiro, v. 24, n. 11, p. 2499-510, 2008.

MARTINS, P. D. Promoção da saúde e bem-estar subjetivo no futebol de formação: regulação emocional e rendimento de atletas acolhidos na Casa do Dragão. 2015. Dissertação (Mestrado em de Mestrado em Psicologia Clínica e da Saúde) - Universidade Portucalense, Porto, 2015.

MENDES NETTO, R. S.; SILVA, C. S.; COSTA, D.; RAPOSO, O. F. F. Nível de atividade física e qualidade de vida de estudantes universitários da área de saúde. Revista Brasileira de Ciências da Saúde, São Paulo, v. 10, n. 34, p. 47-55, 2013.

MOREIRA, D. P.; FUREGATO, A. R. F. Stress and depression among students of the last semester in two nursing courses. Revista Latino-Americana de Enfermagem, São Paulo, v. 21, n. Esp., p. 155-62, 2013.

NORI, R.; GIUSBERT, F. Predicting cognitive styles from spatial abilities. American Journal of Psychology, Austin, v. 119, n. 119, p. 67-86, 2006.

PALMA A. Atividade física, processo saúde-doença e condições sócio-econômicas: uma revisão da literatura. Revista Paulista de Educação Física, São Paulo, v. 14, n. 1, p. 97-106. 2017.

PEKMEZOVIC, T.; POPOVIC, A.; TEPAVCEVIC, D. K.; GAZIBARA, T.; PAUNIC, M. Factors associated with health-related quality of life among Belgrade university students. Quality of Life Research, Oxford, v. 20, n. 3, p. 391-397, 2011.

REIS, M. C.; ELZO JÚNIOR, E. P. P.; MELO, N. S. A.; RAPOSO, M. T.; MUNARO, H. L. R. Condições de saúde e fatores associados a satisfação com vida em acadêmicos de fisioterapia. Saúde.com, Jequié, v. 12, n. 3, p. 636-45, 2016.

RIGO, M. L. N. R.; TEIXEIRA, D. C. Efeitos da atividade física na percepção de bem-estar de idosas que residem sozinhas e acompanhadas. Journal of Health Sciences, Sarajevo, v. 7, n. 1, p. 13-20, 2015.

SILVA, S. G. Caracterização da pesquisa. In: SANTOS, S. G. Métodos de pesquisa quantitativa aplicadas á educação física. Florianópolis: Tribo da Ilha, 2011. p. 67-73.

SILVA, T. R.; SAENGER, G.; PEREIRA, E. F. Fatores associados à imagem corporal em estudantes de educação física. Motriz, Rio Claro, v. 17, n. 4, p. 630-39, 2017.

SOTERO, R. C.; CUNHA, N. C.; MADRID, B.; SALES, M. M.; MOREIRA, S. R.; SIMÕES, H. G. Identificação do lactato mínimo de corredores adolescentes em teste de pista de três estágios incrementais. Revista Brasileira de Medicina do Esporte, São Paulo, v. 17, n. 2, p. 119-22, 2011.

SOUSA, T. F.; SANTOS, S. F. S.; PIE, A. C. S.; ROSSATO, L. C. Associação entre indicadores de prática de atividade física na adolescência com o nível atual de prática de atividade física no lazer em acadêmicos de um curso de educação física no nordeste do Brasil. Pensar a Prática, Goiás, v. 12, n. 3, p. 1-17, 2009.

SPOHR, C.; FORTES, M.; ROMBALDI, A.; HALLAL, P.; AZEVEDO, M. Atividade física e saúde na Educação Física escolar: efetividade de um ano do projeto "Educação Física+". Revista Brasileira de Atividade Física \& Saúde, Pelotas, v. 19, n. 3, p. 300-13, 2014.

WHO. World Health Organization Physical. The use and interpretation of anthropometry. Geneva $\mathrm{CH}$. WHO Technical Report 854, 1995. Disponivel em: <https://www.who.int/childgrowth/publications/ physical status/en/>. Acessado em: 15 de novembro de 2018.

WISÉN, A. G.; FARAZDAGHI, R. G.; WOHLFART, B. A. Novel rating scale to predict maximal exercise capacity. European Journal of Applied Physiology, Berlim, v. 87, n. 4-5, p. 350-57, 2002. 
ZAGATTO, A. M.; PAPOTI, M.; GOBATTO, C. A. Validity of critical frequency test for measuring table tennis aerobic endurance through specific protocol. Journal of Sports Science \& Medicine, Bursa, v. 7, n. 4, p. 461, 2008.

Autor correspondente: Rubian Diego Andrade

E-mail: rubian2@hotmail.com

Recebido: 21 de fevereiro de 2019.

Aceito: 22 de abril de 2019. 